\title{
Lung volume reduction surgery does not increase daily physical activity in patients with severe chronic obstructive pulmonary disease
}

\author{
Noriane A. Sievi ${ }^{1}$, Daniel Franzen ${ }^{1}$, Malcolm Kohler ${ }^{1,2}$, Christian F. Clarenbach ${ }^{1}$ \\ ${ }^{1}$ Division of Pulmonary, University Hospital of Zurich, Zurich, Switzerland; ${ }^{2}$ Zurich Centre for Integrative Human Physiology, University of Zurich, \\ Zurich, Switzerland \\ Contributions: (I) Conception and design: NA Sievi, M Kohler, CF Clarenbach; (II) Administrative support: NA Sievi, CF Clarenbach; (III) Provision \\ of study materials or patients: D Franzen, M Kohler, CF Clarenbach; (IV) Collection and assembly of data: All authors; (V) Data analysis and \\ interpretation: All authors; (VI) Manuscript writing: All authors; (VII) Final approval of manuscript: All authors. \\ Correspondence to: Christian F. Clarenbach, MD. Division of Pulmonary, University Hospital of Zurich, Raemistrasse 100, Zurich 8091, Switzerland. \\ Email: christian.clarenbach@usz.ch.
}

\begin{abstract}
Background: Lung volume reduction surgery (LVRS) is a treatment option for selected patients with severe chronic obstructive pulmonary disease (COPD) and emphysema. The positive effects of LVRS on exercise capacity are well known. In contrast, the effect of LVRS on daily physical activity (PA) is less clear.

Methods: In a prospective case-control study we evaluated selected patients with severe COPD and emphysema who underwent LVRS and COPD patients following usual care. Controls were matched for age, severity of airflow obstruction $\left(\mathrm{FEV}_{1}\right)$ and hyperinflation [residual volume to total lung capacity (RV/TLC)]. Treatment effect of LVRS on activity parameters was analysed using univariable regression model adjusting for treatment group.

Results: A total of 19 patients underwent LVRS and 16 COPD patients without a surgical intervention during the study period were included. The median (quartile) $\mathrm{FEV}_{1} \%_{\text {pred }}$ was $28 \%$ (range, 21-33\%), RV/ TLC was $69 \%$ (range, $64-73 \%$ ) in cases while controls had a median (quartile) $\mathrm{FEV}_{1} \%$ pred of $33 \%$ (range, 28.5-49.5\%) and a RV/TLC of 58\% (range, 49-61\%). Age and body mass index (BMI) were comparable between both groups. Number of steps per day following LVRS was comparable to before the intervention (mean change: $-115,95 \% \mathrm{CI}$ : -994.6 to $764.3, \mathrm{P}=0.779$ ) and was not significantly different to the change in control subjects (mean treatment effect: 931.4 , 95\% CI: -252.4 to 2,115.1, P=0.117).

Conclusions: The results from this study reveal that patients undergoing LVRS did not increase their daily level of PA despite improvement of exercise capacity and symptoms.
\end{abstract}

Keywords: Activity monitor; chronic obstructive pulmonary disease (COPD); exercise capacity; lung volume reduction surgery (LVRS); physical activity level (PAL)

Submitted Oct 27, 2017. Accepted for publication Apr 20, 2018.

doi: $10.21037 /$ jtd.2018.05.18

View this article at: http://dx.doi.org/10.21037/jtd.2018.05.18

\section{Introduction}

Lung volume reduction surgery (LVRS) is a treatment option for patients with severe chronic obstructive pulmonary disease (COPD) and emphysema. This surgical technique helps to improve symptoms by removing the most diseased and non-functioning parts of the lungs.
Usually this patient group is symptomatic despite maximal bronchodilator therapy and optimal medical management including pulmonary rehabilitation. The National Emphysema Treatment Trial (1) defined patients who are likely to benefit from LVRS potentially improving lung function, exercise capacity, dyspnea and quality of 
life. Analysis of long term outcomes after LVRS revealed that improvements in this patient group appear to last approximately 5 years (2).

The positive effects of LVRS on exercise capacity are well known. A recent Cochrane analysis (3) included five randomized controlled trials (215 patients) comparing the effectiveness of LVRS versus non-surgical standard therapy in improving several health outcomes for patients with severe emphysema such as mortality and quality of life. The results demonstrated that the 6 minutes walking distance (6MWD) improved on average by 70 meters after LVRS. However, despite improvement of exercise capacity the effect of lung volume reduction (i.e., coils, valves and surgery) on daily physical activity (PA) is less clear (4-6). Preserved PA has been associated with reduced risk of hospitalizations and mortality in patients with COPD (7). LVRS provides a substantial improvement of airflow obstruction and a reduction of hyperinflation. As hyperinflation has been previously identified as an independent predictor of daily PA (8), it could be assumed that LVRS also leads to an improvement of activity.

In the present study, our objective was to evaluate the impact of LVRS on daily PA in patients with severe COPD and to compare the results to patients following usual care in a case-control study. We hypothesized that LVRS will lead to an increase in objectively assessed daily PA in COPD.

\section{Methods}

\section{Subjects}

The Obstructive Pulmonary Disease Outcomes Cohort Study (TOPDOCS) is a prospective, non-interventional cohort project including mild to very severe COPD patients from seven pulmonary outpatient clinics in Switzerland with annual assessments. Patients with COPD according to global initiative for chronic obstructive lung disease (GOLD) guidelines, aged between 40 and 75 years at inclusion were recruited during outpatient visits or during hospital (9) stay between October 2010 and April 2016. In case of an exacerbation patients were included into the study with a delay of at least 6 weeks. Selected patients with severe COPD and emphysema underwent LVRS while being part of this cohort study. In the current sub-study, these cases were matched to control subjects with severe COPD following usual care in this cohort. Controls were matched for age, severity of airflow obstruction [(forced expiratory volume in 1 second $\left.\left(\mathrm{FEV}_{1}\right)\right]$ and hyperinflation [(residual volume to total long capacity (RV/TLC)]. Since most of the LVRS patients received pulmonary rehabilitation in the time period following surgery, only control subjects receiving pulmonary rehabilitation within the study period were included. The study was conducted in accordance with the declaration of Helsinki and all subjects gave written informed consent to participate. The Ethics Committee of the Canton of Zurich approved the study (EK-ZH-NR: 2011-0106) and the study is registered at www.ClinicalTrials.gov, NCT01527773.

\section{Measurements}

\section{Exercise capacity}

Exercise capacity was assessed by the 6-minute-walk test that was performed according to American Thoracic Society guidelines (10).

The 1-min sit-to-stand (1-min STS) test and handgrip strength test have been proposed as simple tests of exercise performance in COPD (11). Both tests were performed according to standardized protocols (12).

\section{Daily PA}

Performance-based daily PA (number of steps per $24 \mathrm{~h}$ ) and physical activity level (PAL) were assessed using a multisensory armband (SenseWear-Pro armband; Bodymedia, Pittsburgh, PA, USA), which was worn on the upper arm for 7 consecutive days. The device is suited to assess physical activities in COPD patients (13). The threshold for valid data from the armband was set at 4 days with a minimum of 22.5 hours/day. Different levels of PA were defined according the recommendation from the American College of Sports Medicine and the American Heart Association as inactive [(metabolic equivalent of task $($ MET) $<3)$, moderate active (MET: 3-6) and active $(\mathrm{MET}>6)](14)$.

\section{Respiratory variables}

All subjects underwent standard pulmonary functional testing according to ATS/ERS guidelines $(15,16)$ to measure $\mathrm{FEV}_{1}$, forced vital capacity (FVC), RV/TLC and diffusing capacity for carbon monoxide (DLCO). Only values after bronchodilation are shown.

Daytime arterial oxygen saturation $\left(\mathrm{SaO}_{2}\right)$ was measured by arterial blood gas analysis (ABL 700 series, Radiometer Copenhagen) after 5 minutes of rest. 


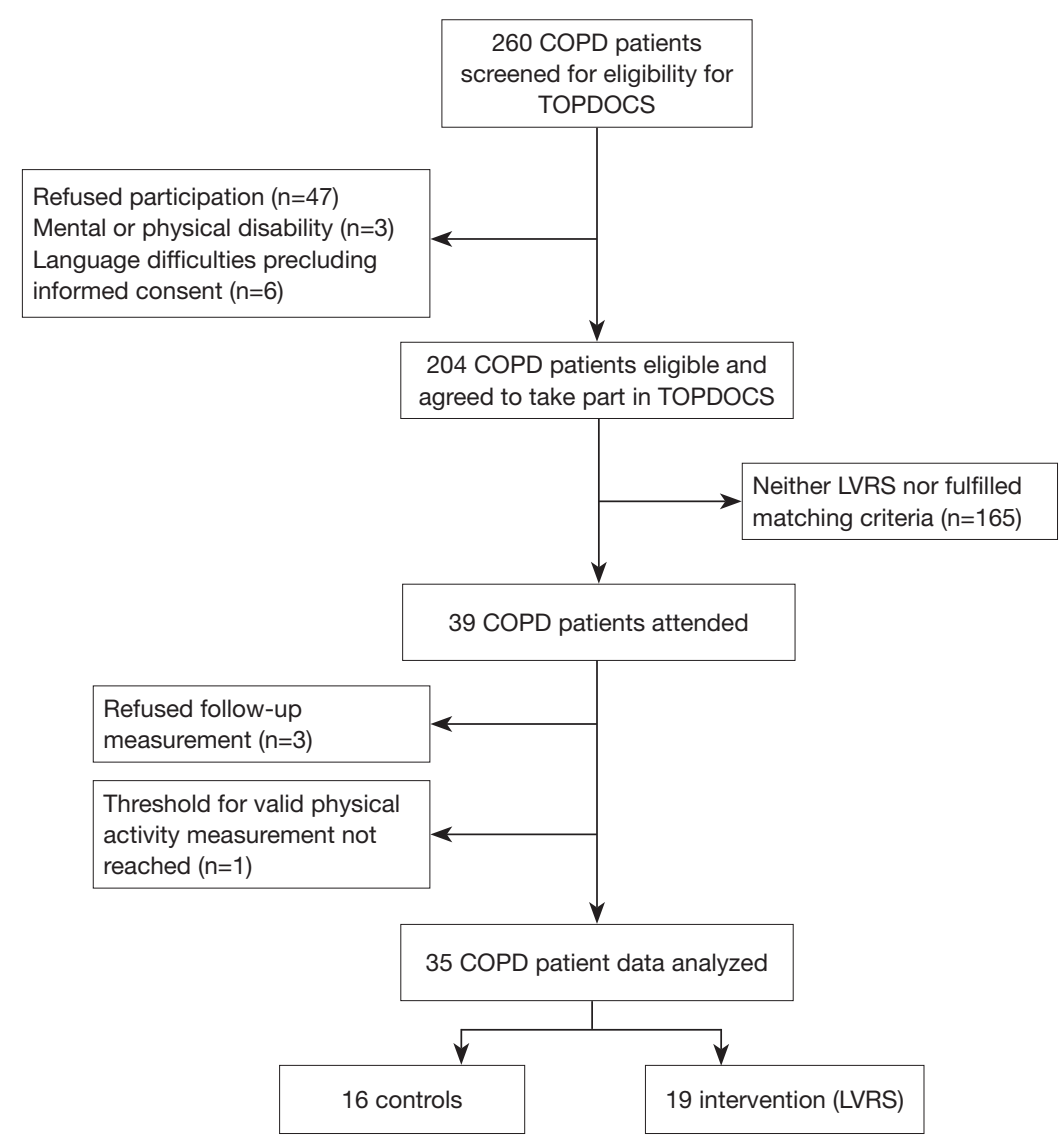

Figure 1 Study flow. COPD, chronic obstructive pulmonary disease; TOPDOCS, Obstructive Pulmonary Disease Outcomes Cohort Study; LVRS, lung volume reduction surgery.

\section{Data analysis and statistics}

All results are shown as mean values [standard deviation (SD)] or median (lower and upper quartile) unless otherwise stated. Statistical analysis was performed with STATA 14 (StataCorp, Texas, USA). Missing values were not replaced. Baseline parameters were analysed using unpaired $t$-test for parametric variables and Wilcoxon rank sum test for nonparametric variables. The change in each outcome was analysed using paired $t$-test for parametric and Wilcoxon signed ranks test for non-parametric variables. Treatment effect of LVRS on activity parameters was analysed using univariate regression models. A post-hoc analysis using a multivariate regression model was performed to adjust the treatment effect of LVRS for imbalances in baseline characteristics (6MWD). A two-sided $\mathrm{P}$ value of $<0.05$ was considered to be statistically significant.

\section{Results}

\section{Study participants}

Of the 204 patients who were part of the longitudinal follow-up of the TOPDOCS cohort study, 19 underwent LVRS and were included in this analysis (Figure 1). These patients were compared to 16 COPD patients without a LVRS during the study period. The median (quartiles) age was 65 years (range, 59-68 years), body mass index (BMI) was $22.3 \mathrm{~kg} / \mathrm{m}^{2}$ (range, $20.8-26.7 \mathrm{~kg} / \mathrm{m}^{2}$ ) in cases while controls had a median (quartiles) age of 64 years (range, $61-$ 66 years) and a BMI of $26.2 \mathrm{~kg} / \mathrm{m}^{2}$ (range, $23.7-29.7 \mathrm{~kg} / \mathrm{m}^{2}$ ). Lung function was comparable between both groups [median (quartiles) $\mathrm{FEV}_{1} \%_{\text {pred }} 28 \%$ (range, $21.0-33.0 \%$ ) in LVRS vs. 33\% (range, 28.5-49.5\%) in controls, median (quartiles) RV/TLC 69\% (range, 64-73\%) in LVRS vs. $58 \%$ (range, $49-61 \%$ ) in controls]. The detailed patient 
Table 1 Baseline characteristics

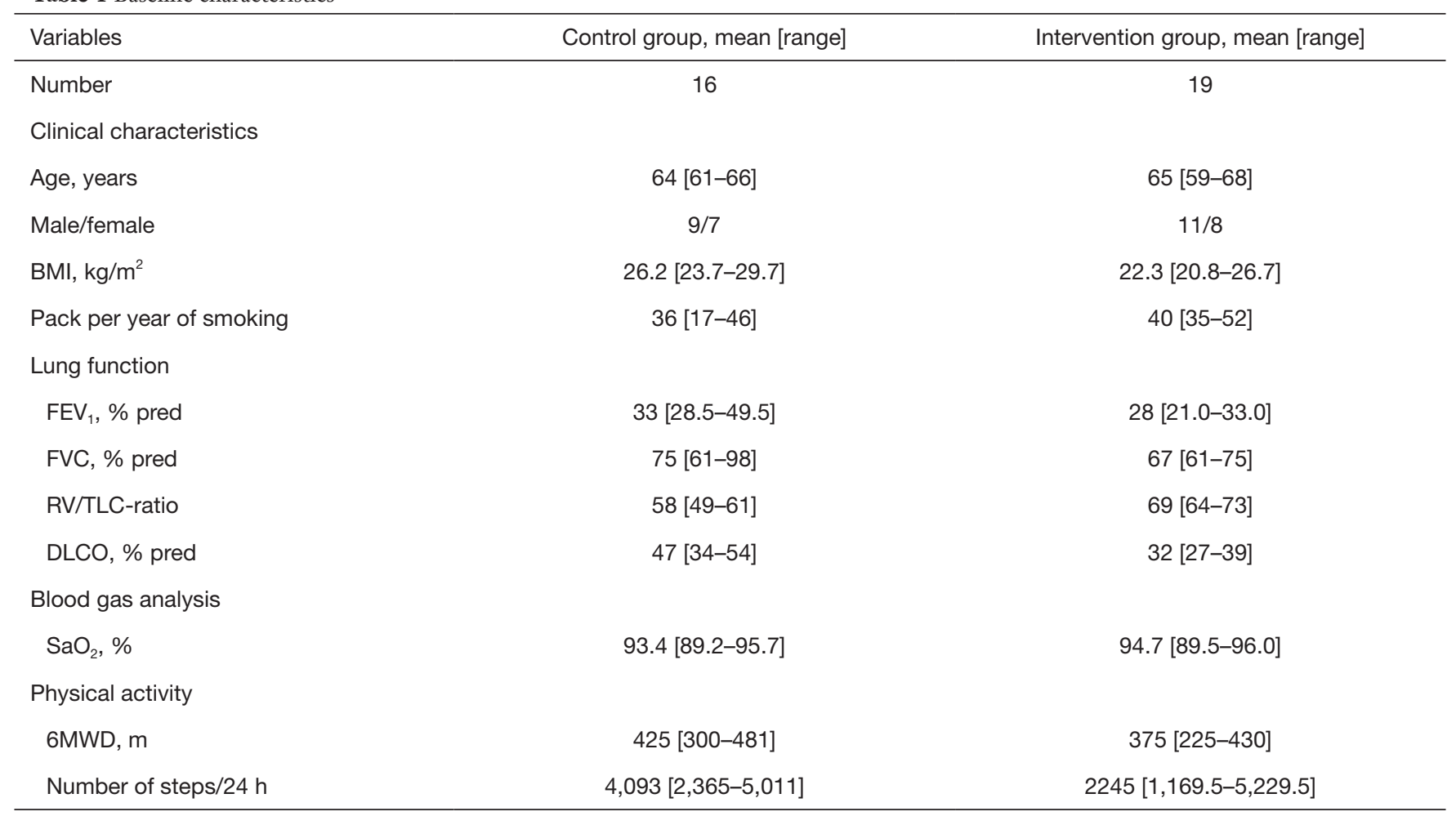

$\mathrm{FEV}_{1}$, forced expiratory volume in 1 second; FVC, forced vital capacity; RV/TLC, residual volume to total lung capacity; DLCO, diffusing capacity for carbon monoxide; $\mathrm{SaO}_{2}$, arterial oxygen saturation; $\mathrm{BMI}$, body mass index; 6MWD, 6-minutes walking distance.

characteristics are shown in Table 1. The follow-up assessment to study the effect of LVRS was performed with a median of 7.3 months (range, 1.6-22.2 months) after LVRS with maintaining the time frame of 1 year between two study visits. The time between pulmonary rehabilitation and follow-up visit was comparable in both groups $(\mathrm{P}=0.868)$.

\section{$P A$ and exercise performance at baseline}

At baseline, median (quartile) number of steps per day was 2,245 (range, $1,169.5-5,229.5$ ) in the LVRS group and 4,093 (range, 2,365-5,011) in the control group ( $\mathrm{P}=0.391$ ). Furthermore, there was no significant difference in all tested baseline activity parameters between the two groups (Table 2).

\section{Change in $P A$}

Table 3 shows the changes of all measured PA parameters in subjects who underwent LVRS and matched controls. The number of steps per day following LVRS was comparable to the number of steps per day before the intervention (median change: $-115.2,95 \%$ CI: -994.6 to 764.3 ). The decrease in steps per day in control subjects (median change: $-1,047$, $95 \%$ CI: $-1,934.7$ to -158.4 ) was not significant. Moreover, there was no significant mean treatment effect of 931.4 (95\% CI: -252.4 to $2,115.1)$ steps per day $(\mathrm{P}=0.117)$, remaining non-significant after adjustment for baseline number of steps per day. As in the control group, the percentage of time spent at different levels of MET (inactive/moderate active/active) did not significantly change between baseline and follow up in the intervention group (Figure 2).

\section{Change in exercise capacity}

In the intervention group 6MWD significantly enhanced at follow-up assessment $(\mathrm{P}=0.017)$ while the control group showed no significant changes. Furthermore, change in $6 \mathrm{MWD}$ adjusted for baseline walking distance remained significant in the LVRS group $(\mathrm{P}=0.049)$. Mean treatment effect was significant with 68.3 (95\% CI: 14.3-122.3; 
Table 2 Comparison of activity parameters at baseline

\begin{tabular}{|c|c|c|c|}
\hline Variables & Control group, mean [range] & Intervention group, mean [range] & $\mathrm{P}$ \\
\hline 6MWD, m & $425[300-481]$ & 375 [225-430] & 0.066 \\
\hline Number of sit to stand & $20[13.5-23.0]$ & $17[14-18]$ & 0.355 \\
\hline Handgrip, kg & 31.7 [24.6-42.8] & $34.8[28.0-39.0]$ & 0.818 \\
\hline Time spent at $>3 \mathrm{MET}$, min/day & $13[4.0-45.0]$ & $8[1.0-41.5]$ & 0.486 \\
\hline Time spent inactive (<3 MET), min/day & $1,397[1,379-1,426]$ & $1,407[1,387-1,421.5]$ & 0.948 \\
\hline Time spent moderate active (3-6 MET), min/day & $12[4.0-45.0]$ & $8[1.0-36.5]$ & 0.536 \\
\hline Time spent active (>6 MET), min/day & $0[0.0-2.0]$ & $0[0.0-0.5]$ & 0.509 \\
\hline
\end{tabular}

MET, metabolic equivalent of task; 6MWD, 6-minutes walking distance.

$\mathrm{P}=0.015)$.

In both groups, number of sit to stand and handgrip as further variables of exercise capacity did not change between baseline and follow-up (Table 3).

\section{Change in lung function}

LVRS led to a mean increase in $\mathrm{FEV}_{1} \%_{\text {pred }}$ of $7.5 \%$ (SD: $1.6 \%$ ) and RV/TLC decreased by $-8.9 \%$ (SD: $2.0 \%$ ).

\section{Discussion}

This case-control study investigated various measures of daily PA and exercise capacity in patients with COPD receiving LVRS, optimal medical therapy and pulmonary rehabilitation as compared to patients only receiving the latter two. The main findings of this study reveal that patients undergoing LVRS did not increase their daily level of PA despite improvement of exercise capacity and lung function.

In patients with severe COPD and end-stage emphysema, LVRS is an option to obtain clinically meaningful improvement in quality of life, lung function and exercise tolerance. However, it is unclear whether these improvements also translate in a higher amount of daily PA. Therefore, we evaluated patients before and after LVRS and compared them to a control group that received optimal medical therapy including pulmonary rehabilitation during the observational period. Control subjects were comparable regarding baseline PA and exercise capacity.
As alternative to LVRS, there are two options of bronchoscopic lung volume reduction (BLVR). Both, lung volume reduction coils (LVRC) and endobronchial valves (EBV) have been demonstrated to improve exercise capacity, symptoms and lung function as recently summarized by Shah et al. (17).

A previous study investigated the effect of LVRC for severe emphysema on activity pattern (5). LVRCs are implanted bronchoscopically in their straightened form and revert to their original double loop shape after lobar placement to achieve mechanical volume reduction. Fourteen patients with a baseline number of steps per day of 2,084 were investigated before and 6 months after the BLVR. After 6 months, no significant changes in PA were detected, despite significant improvement of lung hyperinflation. In contrast, a study by the same group recently performed in 19 patients receiving EBV treatment as alternative option to LVRS showed an impressive increase of 1,340 steps per day 6 months after the intervention (6). The change in steps per day was not associated with any other clinical variables such as change in $\mathrm{FEV}_{1}$ and exercise capacity. Compared to the author's first study investigating coils, the baseline number of steps per day was significantly higher (3,456 steps per day). Furthermore, the longitudinal effect of LVRS on daily PA remains to be examined in further studies.

Demeyer et al. (18) investigated the minimal important difference (MID) in PA in patients with COPD. Daily PA was measured in 74 patients before and after 3 months of pulmonary rehabilitation. The results of this study showed 


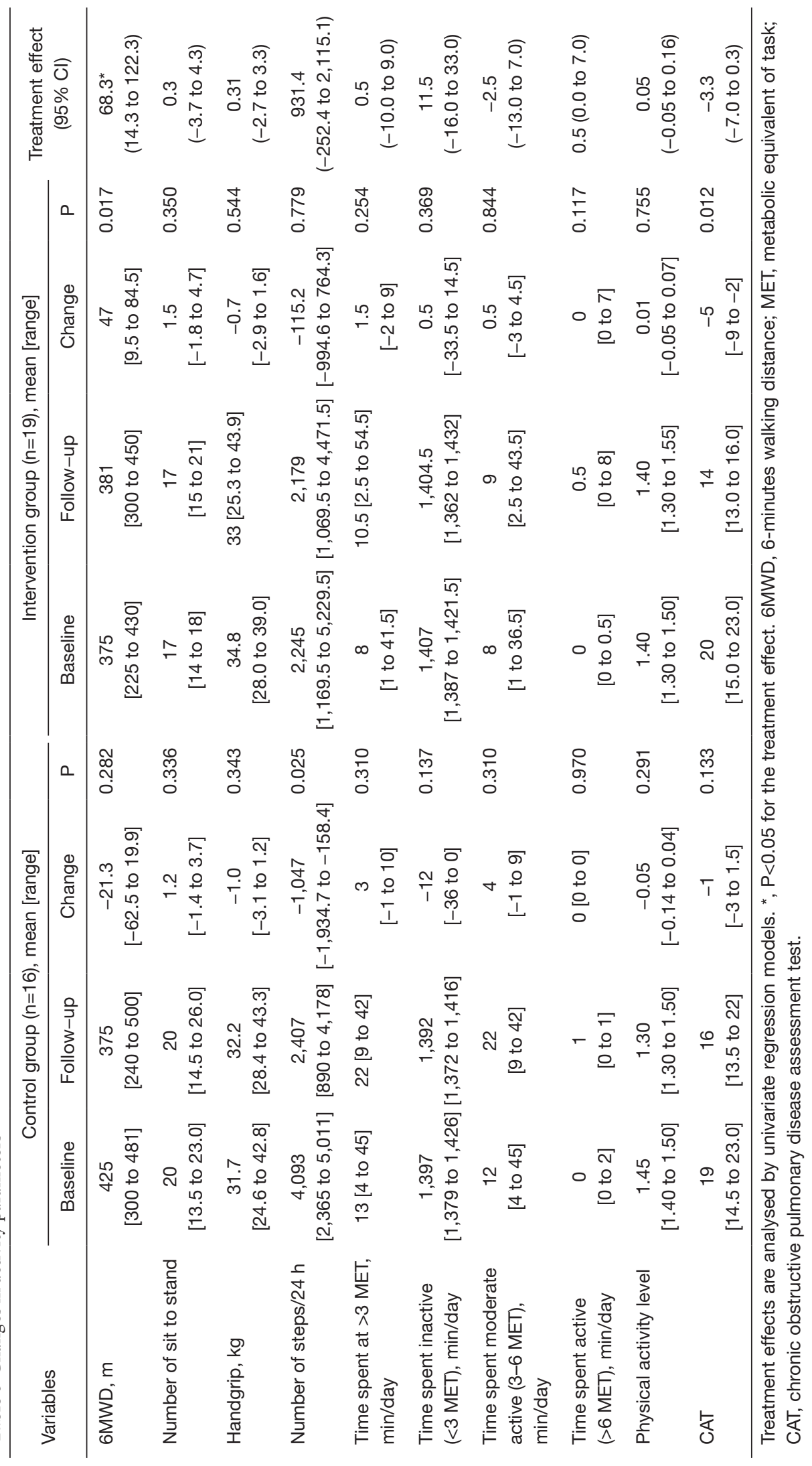



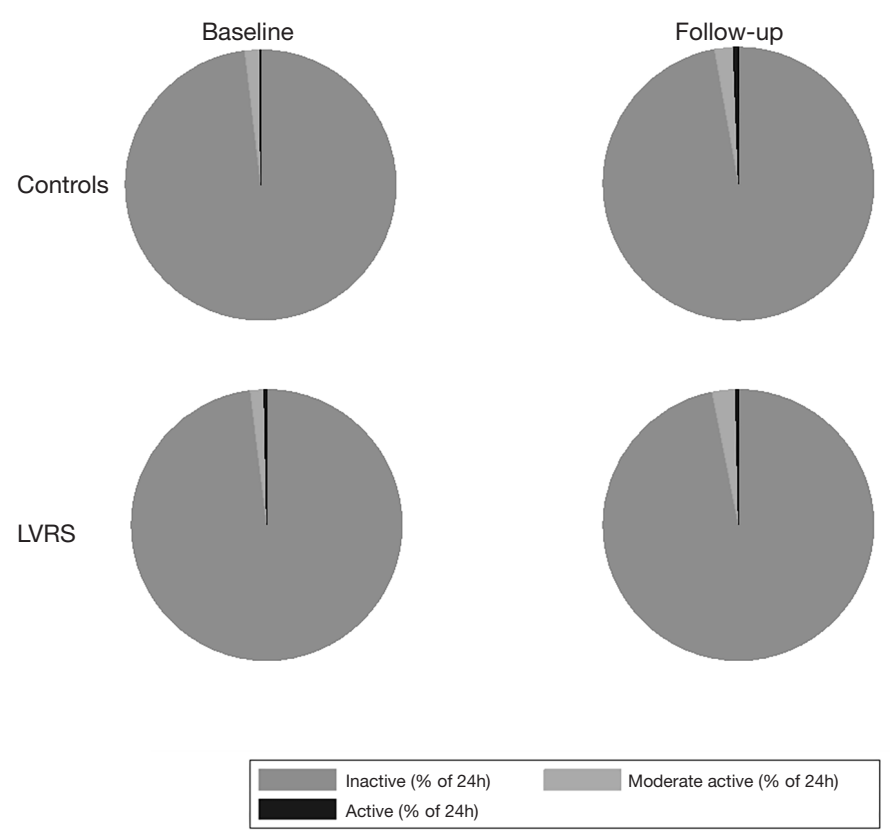

Figure 2 The figure illustrates the percentage of time spent at different levels of PA (inactive/moderate active/active) in both groups at baseline and follow up. The allocation did not significantly change between baseline and follow up and the changes were comparable between both groups (inactive $\mathrm{P}=0.327$, moderate active $\mathrm{P}=0.550$, active $\mathrm{P}=0.218$ ). LVRS, lung volume reduction surgery; $\mathrm{PA}$, physical activity.

that the MID lies between 600 and 1,100 steps per day. In our study, neither the intervention group nor the control group enhanced their PA at follow-up visit. In contrast to the study by Demeyer et al., we performed the follow-up visit with a mean of 6 months after rehabilitation. However, since most of the studies assessing the effect of pulmonary rehabilitation on $\mathrm{PA}$ end 3 months after rehabilitation it is still unclear if there is a sustained effect on activity behaviour (19). In this context a previous review on the effect of exercise training on daily PA showed a small increase of PA after rehabilitation, however, a clinically meaningful change of activity behaviour was not achieved (20).

Although exercise capacity and symptoms are still meaningful increased 6 months after LVRS, this potential benefit does not translate into a change of activity behaviour. We speculate that lung volume reduction without modification of patient behaviour including motivational and psychological aspects is unlikely to be sufficient to change the actual amount of daily PA that a patient engages in.

Another approach in this field is not to focus on an increase in time in moderate-to-vigorous intense activities after interventions but on enhancing time spent for leisure activities or domestic household activities (21). However, the results of our study showed no change in time spent at different activity levels (inactive/moderate active/active) after LVRS. Patients in the intervention and control group maintained their usual activity behaviour although their exercise capacity was enhanced after LVRS. Furthermore, symptoms assessed by COPD assessment test (CAT) were reduced above the MID of two points (22). The findings on exercise capacity and symptoms are consistent with previous findings reported in patients with severe COPD undergoing LVRS. The intervention group increased their mean $(95 \%$ CI) $6 \mathrm{MWD}$ by 68.3 meters (range, $14.3-122.3$ meters). This change is evident above the MID for 6MWD in COPD of 25 meters (23).

The study was conducted as part of the TOPDOCS cohort study and therefore the sample size calculation was not performed for this outcome. We cannot exclude that our findings may be due to a small sample size. However, our findings indicate that LVRS does not lead to a clinically meaningful increase in daily PA. Furthermore, lung function, symptoms and exercise capacity changed after LVRS above the MID pointing towards a better health condition after LVRS. 


\section{Conclusions}

The results from our study reveal that patients undergoing LVRS did not increase their daily level of PA despite improvement of lung function, exercise capacity and symptoms.

\section{Acknowledgements}

This study was supported by Lunge Zurich.

\section{Footnote}

Conflicts of Interest: The authors have no conflicts of interest to declare.

Ethical Statement: The study was approved by the Ethics Committee of the Canton of Zurich (EK-ZH-NR: 20110106) and written informed consent was obtained from all patients.

\section{References}

1. Fishman A, Martinez F, Naumheim K, et al. A randomized trial comparing lung-volume-reduction surgery with medical therapy for severe emphysema. $\mathrm{N}$ Engl J Med 2003;348:2059-73.

2. Ciccone AM, Meyers BF, Guthrie TJ, et al. Longterm outcome of bilateral lung volume reduction in 250 consecutive patients with emphysema. J Thorac Cardiovasc Surg 2003;125:513-25.

3. van Agteren JE, Carson KV, Tiong LU, et al. Lung volume reduction surgery for diffuse emphysema. Cochrane Database Syst Rev 2016;10:CD001001.

4. Clarenbach CF, Sievi NA, Brock M, et al. Lung Volume Reduction Surgery and Improvement of Endothelial Function and Blood Pressure in Patients with Chronic Obstructive Pulmonary Disease. A Randomized Controlled Trial. Am J Respir Crit Care Med 2015;192:307-14.

5. Hartman JE, Boezen HM, Heintzbergen S, et al. Daily physical activity after bronchoscopic lung volume reduction: a pilot study. Eur Respir J 2012;40:1566-7.

6. Hartman JE, Klooster K, Slebos DJ, et al. Improvement of physical activity after endobronchial valve treatment in emphysema patients. Respir Med 2016;117:116-21.

7. Garcia-Aymerich J, Lange P, Benet M, et al. Regular physical activity reduces hospital admission and mortality in chronic obstructive pulmonary disease: a population based cohort study. Thorax 2006;61:772-8.

8. Garcia-Rio F, Lores V, Mediano O, et al. Daily physical activity in patients with chronic obstructive pulmonary disease is mainly associated with dynamic hyperinflation. Am J Respir Crit Care Med 2009;180:506-12.

9. Rabe KF, Hurd S, Anzueto A, et al. Global strategy for the diagnosis, management, and prevention of chronic obstructive pulmonary disease - GOLD executive summary. Am J Respir Crit Care Med 2007;176:532-55.

10. Brooks D, Solway S, Gibbons WJ. ATS statement on six-minute walk test. Am J Respir Crit Care Med 2003;167:1287.

11. Crook S, Frei A, Ter Riet G, et al. Prediction of longterm clinical outcomes using simple functional exercise performance tests in patients with COPD: a 5-year prospective cohort study. Respir Res 2017;18:112.

12. Puhan MA, Siebeling L, Zoller M, et al. Simple functional performance tests and mortality in COPD. Eur Respir J 2013;42:956-63.

13. Van Remoortel H, Raste Y, Louvaris Z, et al. Validity of six activity monitors in chronic obstructive pulmonary disease: a comparison with indirect calorimetry. PLoS One 2012;7:e39198.

14. Haskell WL, Lee IM, Pate RR, et al. Physical activity and public health: updated recommendation for adults from the American College of Sports Medicine and the American Heart Association. Med Sci Sports Exerc 2007;39:1423-34.

15. Miller MR, Hankinson J, Brusasco V, et al. Standardisation of spirometry. Eur Respir J 2005;26:319-38.

16. Macintyre N, Crapo RO, Viegi G, et al. Standardisation of the single-breath determination of carbon monoxide uptake in the lung. Eur Respir J 2005;26:720-35.

17. Shah PL, Herth FJ, van Geffen WH, et al. Lung volume reduction for emphysema. Lancet Respir Med 2017;5:147-56.

18. Demeyer H, Burtin C, Hornikx M, et al. The Minimal Important Difference in Physical Activity in Patients with COPD. PLoS One 2016;11:e0154587.

19. Mantoani LC, Rubio N, McKinstry B, et al. Interventions to modify physical activity in patients with COPD: a systematic review. Eur Respir J 2016;48:69-81.

20. Cindy Ng LW, Mackney J, Jenkins S, et al. Does exercise training change physical activity in people with COPD? A systematic review and meta-analysis. Chron Respir Dis 2012;9:17-26.

21. Sewell L, Singh SJ, Williams JE, et al. Can individualized rehabilitation improve functional independence in elderly patients with COPD? Chest 2005;128:1194-200. 
22. Kon SS, Canavan JL, Jones SE, et al. Minimum clinically important difference for the COPD Assessment Test: a prospective analysis. Lancet Respir Med 2014;2:195-203.

23. Holland AE, Hill CJ, Rasekaba T, et al. Updating the

Cite this article as: Sievi NA, Franzen D, Kohler M, Clarenbach CF. Lung volume reduction surgery does not increase daily physical activity in patients with severe chronic obstructive pulmonary disease. J Thorac Dis 2018;10(5):2722-2730. doi: $10.21037 /$ jtd.2018.05.18 minimal important difference for six-minute walk distance in patients with chronic obstructive pulmonary disease. Arch Phys Med Rehabil 2010;91:221-5. 